\title{
ADVANCED CRUISE MISSILE GUIDANCE SYSTEM DESCRIPTION
}

\author{
Sue Hicks \\ Advanced Cruise Missile SPO \\ ASD/VCE \\ Wright Patterson AFB, OH 45433-6503
}

\begin{abstract}
:
This paper will provide a functional description of the navigation and guidance system in the Advanced Cruise Missile (ACM), one of the Air Force's newest stealth missiles. It will also discuss some of the areas of improvement over the Air Launched Cruise Missile (ALCM). The Kalman Filter design accommodates velocity updates, including lateral velocities, and a nominal number of position updates from Terrain Correlation Matching (TERCOM) to achieve a high accuracy, long range mission.
\end{abstract}

\section{INTRODUCTION:}

The Advanced Cruise Missile System Program Office is located at Wright Patterson Air Force Base Dayton, OH. The Prime Development Contractor is General Dynamics - Convair Division, San Diego California. The Dual Production Contractor is McDonnell Douglas Missile Systems Company, Titusville Florida. The Guidance Set $(G / S)$ is a derivative of the F16 's and B-1B's Navigation Units and was designed and produced by Kearfott Guidance and Navigation Division - A Subsidiary of Astronautics Corporation of America. The Laser Doppler Velocimeter (LDV) was designed specifically for the
ACM and is built by Hughes Aircraft Company - El Segundo.

The Advanced Cruise Missile (ACM) is the Air Force's newest stealth missile. The ACM offers many improvements over the Air Launched Cruise Missile (ALCM). The ACM provides longer standoff and total mission range, requires fewer position updates, and achieves a higher accuracy at the target than the ALCM. The ACM's stealth characteristics improves its survivability as well. The ACM successfully achieved or exceeded its navigation requirements in the flight test program, which was completed in July 1990.

\section{MISSION DESCRIPTION:}

The ACM is carried externally on the B-52H, up to six per pylon. The ACM is launched from great standoff range, at which time the wings and fins deploy and the LDV is powered on. The vehicle passes at subsonic speed through several timing control points along its preprogrammed course. As specified in the mission plan, the ACM performs terrain following during designated portions of the mission. The navigation system is updated with velocity information from the LDV per the mission plan. In a typical mission, 
the ACM takes it position updates from four TERCOM maps - a landfall map, two enroute maps and a terminal map. It navigates to the target where it detonates at a preprogrammed Height Of Burst (HOB). A generic mission is shown in figure 1.

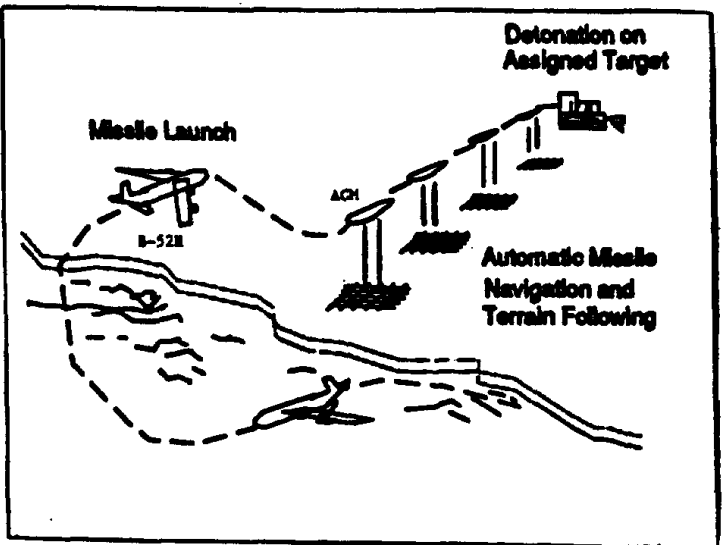

Figure 1 - ACM Mission Profile

The carrier provides initialization, alignment, and mission profile data to the guidance set via the Umbilical Assembly (UA) during captive carry. The data blocks from the carrier that the ACM uses are the same as those used by the ALCM and SRAM. Upon separation, the guidance set processes steering and engine control commands to navigate along the mission route to the target.

The ACM is capable of performing missions that are terrain aided, i.e. TERCOM updating is utilized, or free inertial. The mission can be flex targeted or direct targeted. In a flex targeted mission, the carrier aircraft redefines a new target by specifying a new target location that is on a straight line from any existing waypoint. In a direct targeted mission, the
Carrier Aircraft Equipment (CAE) programs the air vehicle to fly directly from a launch point to a target without use of any waypoints or TERCOM updates. The guidance set memory can accommodate up to 192 waypoints and 10 TERCOM map areas.

\section{GUIDANCE SET:}

The guidance set $(\mathrm{G} / \mathrm{S})$ consists of five functional components - the Inertial Reference Unit (IRU), Air Vehicle Digital Computer Unit (ADCU), Rate and Acceleration Sensor (RAS), and Power Supply Unit (PSU). The guidance set interfaces and communicates with the air vehicle avionics and the carrier aircraft.

The navigation function is performed by the Inertial Reference Unit (IRU) and its supporting electronics which reside on the Platform Electronics cards (1 and 2) and the Gyro Wheel Supply card. The inertial platform is a four gimbaled system with two double axis gyros, the vertical gyro and the azimuth gyro, and three orthogonally mounted accelerometers.

The Air Vehicle Digital Computer Unit (ADCU) is the main processor of all the navigation and flight control functions, or the "brains" of the system. The ADCU is embedded in the guidance set. Memory consists of $128 \mathrm{~K}$ of RAM and $64 \mathrm{~K}$ of Electrically Erasable Programmable Read Only Memory (EEPROM). The ADCU communicates with $\mathrm{ACM}$ subsystems through direct memory access or MILSTD-1750A instructions. The types of Input/Output are discrete, analog to digital, 
digital to analog, and serial data bus. These functions are performed by the Computer Processing Unit (CPU) card, the Digital I/O card, the Analog to Digital (A/D) card, the Digital to Analog (D/A) card, the Serial I/O card, the Discrete I/O card, and two memory cards. The ADCU's processing speed is $580 \mathrm{~K}$ operations/sec. The ADCU uses EEPROM to act as a non-volatile memory for storing the OFS. At power on, the OFS is copied from EEPROM to RAM for execution. Mission data is stored in RAM only.

The Rate and Acceleration Sensor (RAS) is a module that provides air vehicle body rates and accelerations to the ADCU for flight control functions. The RAS consists of three single axis strapdown gyros and two accelerometers, mounted to sense normal and lateral accelerations. Although roll, pitch, and yaw information could have been derived from the platform data, the platform data rate was too slow to adequately support flight control processing. The flight control commands are output of the guidance set to the Aft Avionics Unit (AAU). The AAU interprets the guidance set commands for the fin actuators.

The Power Supply Unit (PSU) is a new design. It consists of the Input power Conditioner (IPC), the DC/DC card and the $A C / D C$ card. $A C$ and $D C$ power was required to meet system warm-up requirements, thus the AC/DC card was created.

The circuit cards mentioned above and the other guidance set components are shown in Figure 2.

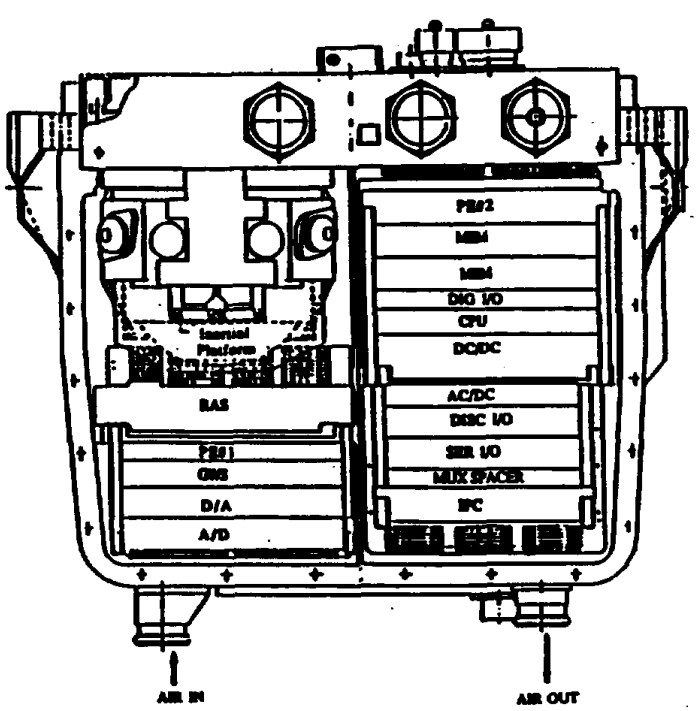

Figure 2 - Top View of Open Guidance Set

The Air Modulator in an air control valve to provide the platform with constant cooling over the temperature/flow range of cooling air from the Environmental Control System (ECS). The air modulator consists of a temperature sensor on the platform case which provides a signal to electronic comparators. A signal that differs from the comparator set point will result in an electronic output to the air valve's drive motor. The drive motor adjusts the valve position to allow the cooling air to return the case to an acceptable operating temperature.

In the first step in the operational sequence, the $G / S$ is initialized in the captive carry mode to stabilize the inertial platform at operating temperature. Then the G/S powers the IRU and starts the alignment process with aid from the Carrier Aircraft Equipment (CAE). The CAE provides alignment commands and data, 
mission profile data, and free flight initialization data. Upon command from the CAE, the $G / S$ performs air vehicle Built In Test (BIT) on itself and other avionics components. BIT results are stored and reported to the carrier aircraft upon request.

Upon separation from the carrier, the $\mathrm{G} / \mathrm{S}$ processes and computes steering and engine control commands for the air vehicle flight control system. In the free flight mode, the $G / S$ provides the guidance and control functions required to guide the air vehicle along preprogrammed flight profiles between precise geographical coordinates. All ACM mission steering and flight control calculations are performed by the Operational Flight Software (OFS).

\section{VELOCIMETER HARDWARE DESCRIPTION:}

The Laser Doppler Velocimeter provides the guidance set with accurate measurements of the air vehicle's ground velocity. The velocity data is used to update the Kalman Filter and therefore reduce the navigation errors. The $\mathrm{LDV}$ is operated only during certain times of the mission. It is hard mounted to the bottom of the guidance set, as shown in figure 3 .

The $\mathrm{LDV}$ is comprised of a $\mathrm{CO} 2$ Laser, beam forming and pointing optics, a detector, and signal processing electronics. The Laser beam is expanded and transmitted through a window in the air vehicle to the ground. Light scattered by the ground is collected by the telescope and focused on the detector. The frequency of this light has been Doppler shifted in proportion to the air vehicle velocity. The Doppler shifted beam mixes on the detector with a portion of the laser beam whose frequency has not been shifted. When these two beams mix, they produce a signal current at the Doppler frequency. The Doppler signal at the detector output is processed by the signal processing electronics. The computed velocity is transmitted to the navigation unit for velocity updating.

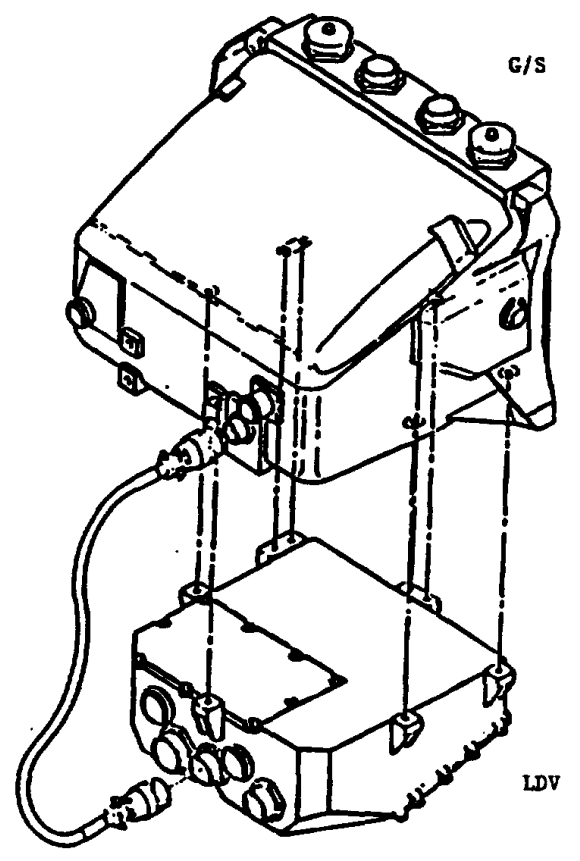

Figure 3 - LDV and G/S Mounting

The actual measurements that are made are the line-of-sight (LOS) component of the ground velocity vector in three non-coplanar directions by detecting the Doppler shift in the frequency of the $\mathrm{CO} 2$ laser beam, and provide the velocity 
information to the $G / S$. Upon command from the guidance set, the LDV will move the beam to one of the three beam positions shown in figure 4. The LDV processes the velocity data to verify its validity of the measurement.

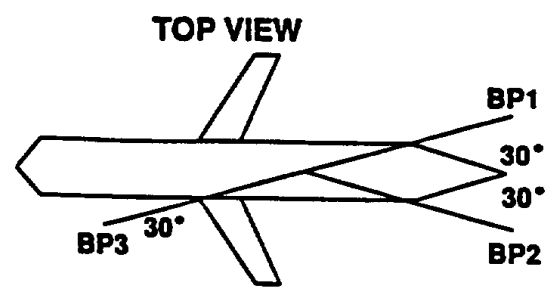

SIDE VIEW

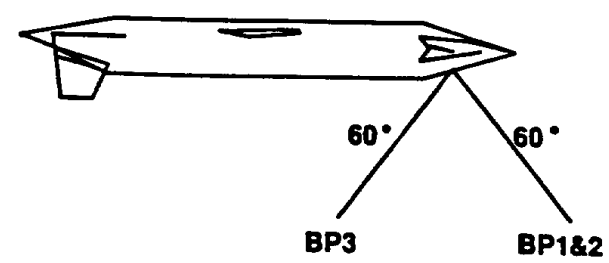

Figure 4 - LDV Beam Positions

The LDV is comprised of five major components: Laser assembly, beam forming optics, pointing optics, detector electronics and signal processing electronics. The functional interface of these components is shown in figure 5.

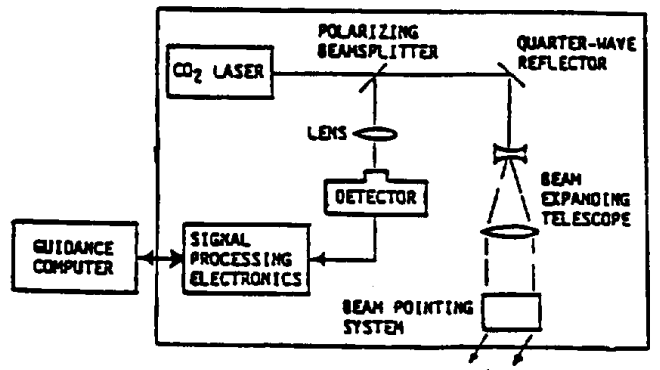

Figure 5 - LDV Functional Interface
The laser is stimulated by an RF power supply. The laser is adjusted to the correct frequency and sent to the beam forming optics, where the laser beam is polarized and shaped. The laser beam is expanded in the pointing assembly and transmitted to the ground through the laser window. Light reflected off the ground is collected by the telescope and focused on the detector. The frequency of the light returned to the detector is Doppler shifted in proportion to the missile ground velocity. At the detector, the Doppler shifted beam is mixed with a portion of the laser beam that has not been shifted. When the two beams mix, they produce a signal at the Doppler frequency. The Surface Acoustic Wave (SAW) processor functions as a spectrum analyzer for this signal and produces an analog signal that contains amplitude vs. frequency information. The signal processor assembly analyzes the SAW output and calculates the velocity in the laser line of sight (LOS). This velocity is then transmitted to the guidance set along with its associated validity. The LDV measures its average LOS velocity over a period of not more than 120 msecs and provides the data to the guidance set at 8 Hz. The LDV meets its performance specification under the condition that the estimated LOS velocity from the guidance set is within $20 \mathrm{ft} / \mathrm{sec}$ of the true LOS velocity.

\section{MISSILE RADAR ALTIMETER:}

The main functions of the Missile Radar Altimeter (MRA) are to measure the missile altitude above ground level and to provide that data to the guidance set for 
Terrain Correlation Matching (TERCOM). The MRA operates over all terrain type. The MRA uses separate transmit and receive antennas in order to increase the altitude range of the system. The guidance set provides control signals to the MRA to enable data transfer and synchronize the data transfer. The MRA transmits altitude data to the guidance set via 16-bit serial words.

\section{KALMAN FILTER:}

The Kalman Filter (KF) processes the CAE position data, TERCOM data and LDV measurement data to correct errors in the level channel navigation. The ACM Kalman Filter is an 18 state filter. The Kalman filter cycle is $12 \mathrm{sec}$. The KF gathers estimates of error sources during alignment using 13 of the 18 states. Error quantities that are used by the Kalman Filter are residual errors, i.e. normal compensation values will be removed before data is sent to the filter. These error source estimates get updated only during LDV aiding or TERCOM updating, else the Kalman Filter deals primarily with process noise. Updating information must meet certain acceptance criteria before the $\mathrm{KF}$ will use the information to update state and covariance matrices. If errors in the system exceed accuracy tolerances, which are typically set at 3 sigma from the expected error value, the Kalman Filter will set a flag that will inhibit launch if in captive carry, and inhibit arming if in free flight. The OFS uses the Kalman Filter estimates for navigation parameters in lieu of the values that are stored in memory from the guidance set's calibration for improved accuracy.

The LDV operates when the mission profile dictates. LDV takes 3 seconds to move the beam into position and gathers data for the remaining 9 seconds of the Kalman Filter cycle. Then the beam is moved to the next position, data is gathered from that position, and so on. LDV data is accumulated at an $8 \mathrm{~Hz}$ rate and a single average measurement is provided to the $\mathrm{KF}$ at the $1 / 12 \mathrm{~Hz}$ rate. LDV data dropouts can occur if the LDV data has been determined to be invalid. The LDV is normally not operating during TERCOM map updating.

The MRA is used to gather altitude AGL data along a column of the TERCOM map. The missile altitude MSL is determined by pressure and temperature data. The terrain height MSL data is stored in memory. By correlating the calculated terrain height with the stored map, the column that the missile is flying over can be determined. The coordinates of the overflown column are used to update the missile's position. This data from the TERCOM update applied back at the start of the map and integrated up. The KF rotates the downtrack and crosstrack errors into the level channel axes, and uses that data to update the state and covariance matrices and the air vehicle direction cosine matrix. As a result of the TERCOM update, the lateral steering function, waypoint management function, and navigation information are improved by the new information.

The MRA is used for vertical updating (VUP) as well for TF and 
TERCOM updating. The number of VUPs performed varies for each mission, however, one VUP is always performed near the target to insure meeting the height of burst (HOB) accuracy requirement. Vertical updating also occurs during TERCOM.

\section{SUMMARY:}

The ACM's navigation and flight control system has made it a versatile and effective weapon system with numerous improvements over existing missile systems. Avionics components were developed to insure stealth characteristics were not adversely impacted. The guidance set met all its performance requirements and exceeded requirements in target accuracy, and timing control. 